\title{
Holographic Optical Data Storage
}

\author{
Doğan A. Timuçin and John D. Downie
}

\section{Historical Introduction}

Although the basic idea may be traced back to the earlier X-ray diffraction studies of Sir W. L. Bragg, the holographic method as we know it was invented by $D$. Gabor in 1948 as a two-step lensless imaging technique to enhance the resolution of electron microscopy, for which he received the 1971 Nobel Prize in physics. The distinctive feature of holography is the recording of the object phase variations that carry the depth information, which is lost in conventional photography where only the intensity (= squared amplitude) distribution of an object is captured. Since all photosensitive media necessarily respond to the intensity incident upon them, an ingenious way had to be found to convert object phase into intensity variations, and Gabor achieved this by introducing a coherent reference wave along with the object wave during exposure. Gabor's in-line recording scheme, however, required the object in question to be largely transmissive, and could provide only marginal image quality due to unwanted terms simultaneously reconstructed along with the desired wavefront. Further handicapped by the lack of a strong coherent light source, optical holography thus seemed fated to remain just another scientific curiosity, until the field was revolutionized in the early 1960 s by some major breakthroughs: the proposition (A. L. Schawlow and C. H. Townes) and demonstration (T. H. Maiman) of the laser principle, the introduction of off-axis holography (E. Leith and J. Upatnieks), and the invention of volume holography (Y. N. Denisyuk). Consequently, the remainder of that decade saw an exponential growth in research on theory, practice, and applications of holography. Today, holography not only boasts a wide variety of scientific and technical applications (e.g., holographic interferometry for strain, vibration, and flow analysis, microscopy and high-resolution imagery, imaging through distorting media, optical interconnects, holographic optical elements, optical neural networks, three-dimensional displays, data storage, etc.), but has become a prominent art, advertising, and security medium as well.

The evolution of holographic optical memories has followed a path not altogether different from holography itself, with several cycles of alternating interest over the past four decades. P. J. van Heerden is widely credited for being the first to elucidate the principles behind holographic data storage in a 1963 paper, predicting bit storage densities on the order of $1 / \lambda^{3}$ with source wavelength $\lambda-\mathbf{a}$ fantastic capacity of nearly $1 \mathrm{~TB} / \mathrm{cm}^{3}$ for visible light! The science and engineering of such a storage paradigm was heavily pursued thereafter, resulting in many novel hologram multiplexing techniques for dense data storage, as well as important advances in holographic recording materials. Ultimately, however, the lack of such enabling technologies as compact laser sources and highperformance optical data $1 / O$ devices dampened the hopes for the development of a commercial product. After a period of relative dormancy, successful applications of holography in other arenas sparked a renewed interest in holographic data storage in the late 1980 s and the early 1990s. Currently, with most of the critical optoelectronic device technologies in place and the quest for an ideal holographic recording medium intensifed, holography is once again considered as one of several future data storage paradigms that may answer our constantly growing need for higher-capacity and faster-access memories.

\section{Holographic Principles}

We show the basic recording and reconstruction arrangements for off-axis holography in Figure 1, assuming that the object whose hologram (meaning "whole record") we wish to make is available in the form of a transparency. Here coherent light from a laser source is collimated to produce a unit-amplitude plane wave normally incident on the object, while at the same time a portion of this plane wave is intercepted by a prism to produce a spatial carrier reference wave (Fig. $1 a$ ). A distance $L$ behind the object is a photosensitive recording medium, which we shall simply refer to as "film" for convenience. The object transparency diffracts, or scatters, the illuminating plane wave, producing across the film plane a complex-amplitude field distribution

$$
O(x, y)=|O(x, y)| e^{i \arg O(x, y)} .
$$

The offset-reference plane wave, meanwhile, is incident on the film at an angle $\theta$ with the $z$ axis, and can be expressed mathematically as

$$
R(x, y)=e^{i t \sin \theta y},
$$




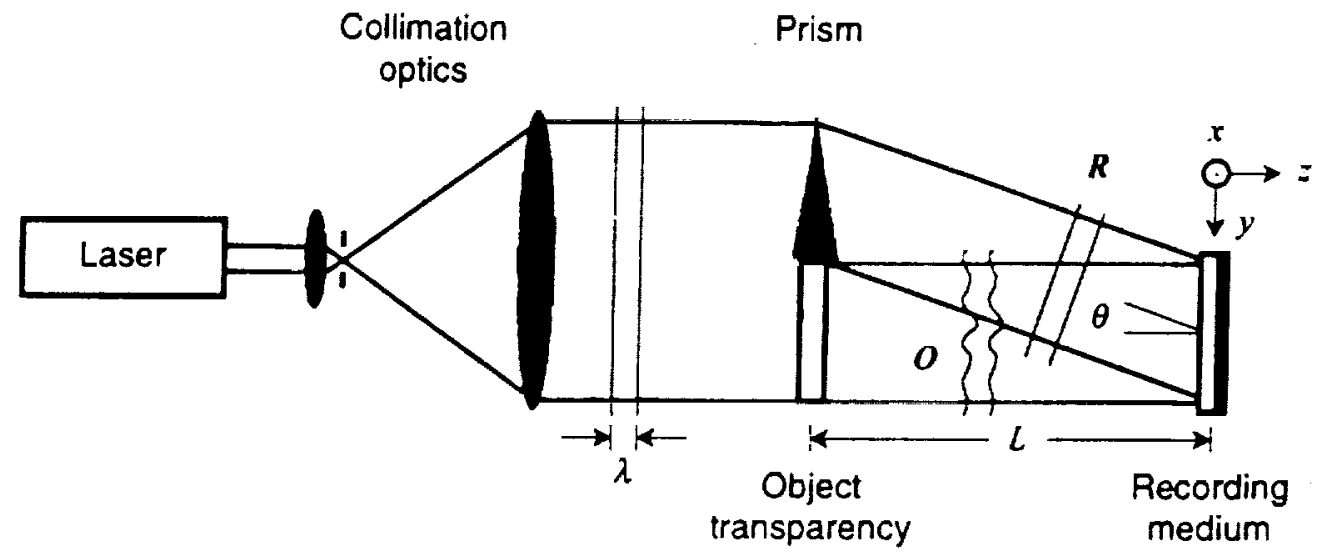

(a) hologram recording

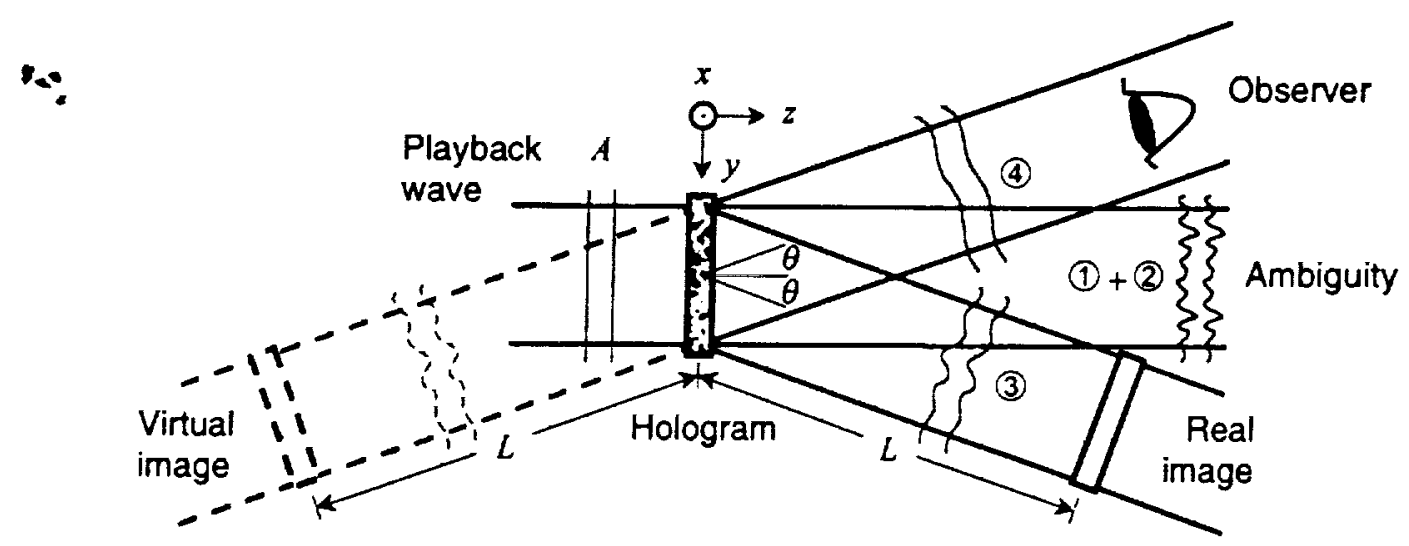

(b) hologram reconstruction

Figure 1 Basic holography - the recording and reconstruction steps for a thin hologram

where $h=2 \pi / \lambda$ is the wave number, and $\lambda$ denotes the source wavelength. These mutually coherent object and reference waves interfere inside the (thin) film, creating the (2-D) intensity distribution

$$
\begin{aligned}
I(x, y) & =|R+O|^{2}=|R|^{2}+|O|^{2}+R O^{\circ}+R^{\circ} O \\
& =1+|O|^{2}+2|O| \cos (k \sin \theta y-\arg O) .
\end{aligned}
$$

Note that the last term of this interference pattern is a standing wave (or "fringe") whose amplitude and phase are modulated by those of the object wave; object phase information has thus been successfully converted to intensity variations inside the film.

Within the linear exposure regime of the photographic medium, the amplitude transmittance of the developed film (i.e., the hologram) becomes

$$
t_{H}(x, y)=t_{0}+\beta_{\varepsilon} I(x, y),
$$

where (bias) $t_{b}$ and (slope) $\beta_{t}$ are (real) constants characteristic of the film and the exposure time $\tau$. If this hologram is now illuminated at normal incidence by a plane wave of amplitude $A$ (Fig. $1 b$ ), then the transmitted field immediately behind the hologram plane is found quite simply to be

$$
\begin{aligned}
U(x, y)= & A t_{H}(x, y) \\
= & A\left(t_{b}+\beta_{\tau}\right)+A \beta_{\tau}|O|^{2} \\
& +A \beta_{z} O^{*} e^{t k \sin \theta y}+A \beta_{z} O e^{-t h \sin \theta y} .
\end{aligned}
$$

The first two terms here are the transmitted plane wave and an ambiguiry field, both of which propagate along the $z$ axis, while the last two terms are encoded on complex-exponential carrier waves and therefore propagate away from the $z$ axis. Specifically, we see that the third term is (up to a constant factor) the complex conjugate of the original object wave, which forms a real (pseudoscopic) image of the object as 
light from the hologram converges in space at a distance $L$ behind the hologram and at an angle $\theta$ with the $z$ axis. Finally, the fourth term is a reconstruction of the original object wavefront, and forms a virtual (orthoscopic) image of the object as an observer sees light from the hologram appear to diverge away from a location a distance $L$ in front of the hologram and at an angle $-\theta$ with the $z$ axis. For faithful reconstruction of the object, it is clearly necessary that these individual terms separate in space as they propagate away from the hologram. One can readily show, with the help of Fourier analysis, that this will indeed be guaranteed if the carrier angle is chosen to satisfy $\theta \geq \arcsin (3 B \lambda)$, where $B$ is the (spatial) bandwidth of the object along the $y$ axis. We thus see that the presence of a suitably chosen spatial carrier reference wave during the recording step is what facilitates the successful subsequent reconstruction of the object from is hologram - an essential feature missing from Gabor's original in-line holography concept and was later introduced by Leith and Upatnieks.

Under a unit-amplitude normally incident planewave illumination, the relationship between the (possibly complex-valued) object amplitude transmittance $t_{v}(\xi, \eta)$ and the recording object wave $O(x, y)$ can be expressed in the form of a linear superposition as

$$
O(x, y)=\iint_{-} K(x, y ; \xi, \eta) t_{0}(\xi, \eta) \mathrm{d} \xi \mathrm{d} \eta
$$

where $K(x, y ; \xi, \eta)$ denotes the propagation kernel between the object and film planes, and is called the point-spread function (or the impulse response) of the intervening optical system. Depending on the particular form of $\boldsymbol{K}$, one can therefore speak of different types of holograms. For instance, if the film falls within the near-field (Fresnel) diffraction region of the object transparency, then the setup of Fig. Ia records what is termed a Fresnel hologram. Now, if a thin positive lens of focal length $f=\frac{1}{2} L$ is inserted halfway between the object and film planes, the corresponding recording is called a Fourier hologram, since the object wave incident on the film in this case is the (2-D) spatial Fourier transform of the object amplitude transmittance. Finally, if a lens with focal length $f=\frac{1}{4} L$ is used instead, then an (inverted) image of the object is formed at the film plane, with the result appropriately called an image hologram.

Fourier holograms provide an excellent misalignment tolerance and make the most efficient use of the hologram space-bandwidth product (i.e., they use a minimal hologram area to record the object information), while image holograms utilize the $d y$ namic range of the recording medium in a much more economical fashion; Fresnel holograms provide a convenient design compromise between these two conflicting requirements. Another advantage of Fourier and Fresnel holograms is the distributed (or redundant) nature of the information storage method that provides robustness against damage: localized defects and degradations in the hologram do not lead to a total loss of recorded information, but merely reduce the signal strength in the retrieved images.

\section{Volume Holograms}

So far we have discussed thin holograms operating in the Raman-ivath diffraction regime whose influence on incident optical waves can simply be characterized by a multiplicative amplitude transmittance function, as we did above. Although images can clearly be stored in and retrieved from such holograms, the true potential of holographic data storage can be realized only when one considers utilizing the third dimension of the recording medium. A grating whose thickness significantly exceeds the fundamental fringe period recorded in it is said to operate in the Bragg diffraction regime, where the extended volume of the medium serves to suppress (or "filter out") all but the first diffraction order in reconstruction. The physics of volume diffraction thus endows the grating with a selectivity property that can be exploited to store data in a multiplexed fashion: many holograms can be stored within the same physical volume and then retrieved independently thanks to a unique addressing scheme, thus greatly enhancing the overall storage capacity of such a medium.

To illustrate the salient features of volume gratings, we consider the basic arrangement shown in Figure 2. (Refraction at the air-medium interfaces, though neglected for clarity in this diagram, is fully accounted for in the following analysis.) Two unitamplitude plane waves of common wavelength $\lambda$ (in air) are incident on the same side of a photosensitive medium of thickness $d$, making angles $\pm \theta$ (in air) with the surface normal (Fig. 2a). (This arrangement records a transmission hologram, whereas incidence from opposite sides of the medium forms a reflection hologram.) For simplicity, the medium is assumed to be transparent (at $\lambda$ ) with an initial refractive index $n$, and a maximum optically induced refractive-index change $\Delta n_{\text {max }}$. The two waves playing the roles of object and reference here may be identified by their wave vectors $\left\{\bar{k}_{o}, \bar{k}_{R}\right\}=k\left(\mp \bar{a}_{y} \sin \theta+\bar{a}_{z} \cos \theta\right)$, and the (3-D) intensity pattern formed by their interference inside the recording medium is then simply

$$
I(\bar{r})=\mid e^{\vec{u}_{n} r}+e^{\left.i \bar{k}_{j} \cdot\right|^{2}}=2\left[1+\cos \left(\vec{k}_{C} \cdot \bar{r}\right)\right]
$$



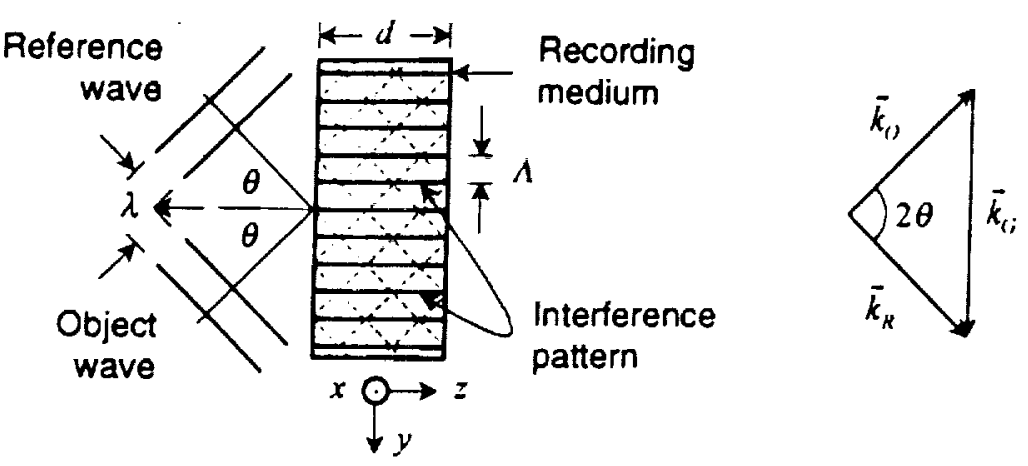

(a) hologram recording

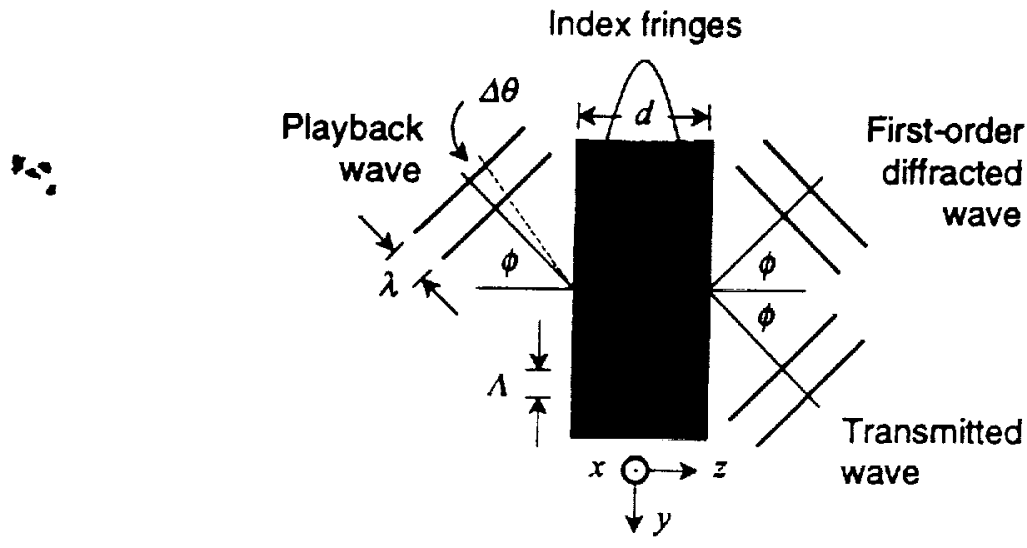

(b) hologram reconstruction

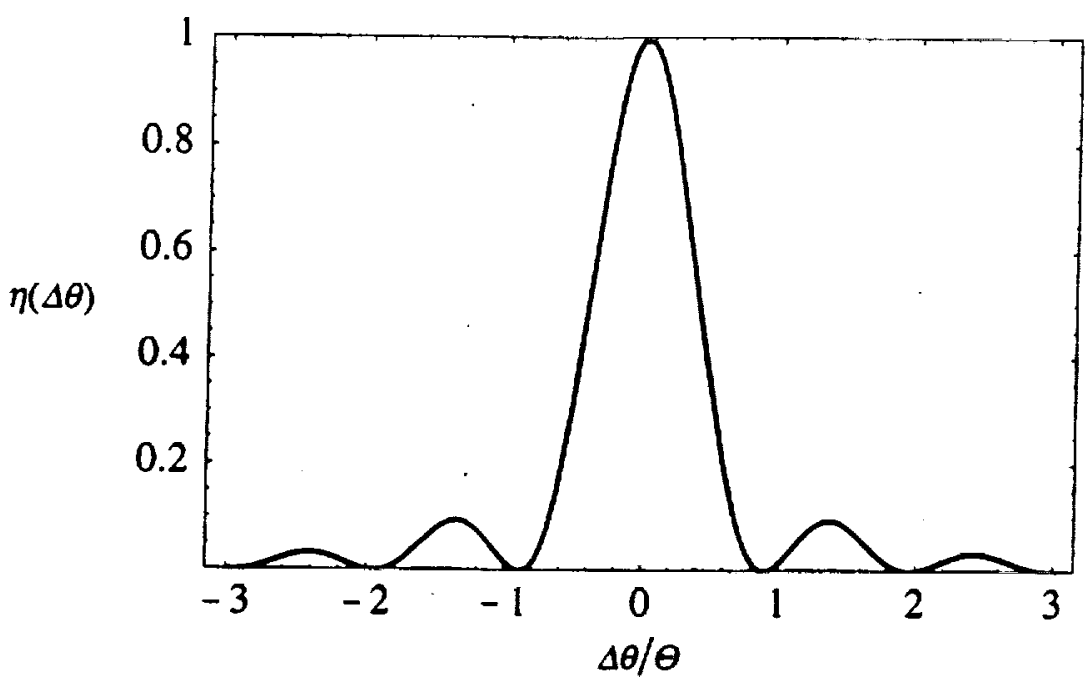

(c) grating angular selectivity

Figure 2 Volume holography - elements of a thick simusoidal phase diffaction grating

Here $\bar{k}_{G}=\bar{k}_{k}-\bar{k}_{o}$ is called the grating vector, and is perpendicular to the intensity fringes (e.g., parallel to the $y$ axis in Fig. $2 a$, with the fringes planes parallel to the $x-z$ plane: $\bar{k}_{c}=\vec{a}_{r} 2 k \sin \theta$ ). We note from the recording wave-vector diagram that the fringe period is $\Lambda=2 \pi /\left|\vec{k}_{G}\right|=\lambda / 2 \sin \theta$. 
The refractive-index distribution inside the medium $(0 \leq z \leq d)$ resulting from this exposure is then

$$
n(\bar{r})=n_{0}+n_{1} \cos \left(\vec{k}_{i j}, \bar{r}\right) \text {. }
$$

assuming an infinite lateral extent. Note that $n_{0} \neq n_{1}$ in general, as the constant background intensity inevitably uses up part of the available dynamic range during exposure. Also, one typically tries to maintain $n_{1} \ll \Delta n_{\max }$ to assure operation in the linear exposure regime and to utilize the material dynamic range economically for multiplexed hologram recording. (The ratio $n_{1} / n_{0}$ is called the modulation transfer function, and represents the spatial frequency response of the recording medium at frequency $\mathrm{l} / \boldsymbol{\Lambda}$.) The transition between the Raman-Nath and Bragg diffraction regimes may be roughly characterized by the parameter $Q \equiv \lambda d / n_{0} \Lambda^{2}:$ a sinusoidal grating is said to be thin if $Q \leq 1$; otherwise it is considered thick.

To reconstruct the object wave, let us now illuminate the grating with a unit-amplitude plane playback wave at the recording wavelength $\lambda$ and at an angle $\phi$ (Fig. $2 b$ ); that is, the playback wave vector is $\bar{k}_{p}=k\left(\bar{a}_{\gamma} \sin \phi+\bar{a}_{z} \cos \phi\right)$. We can develop an intuitive understanding of the volume diffraction process by thinking of the recorded fringe planes as partially reflecting mirrors. (This is literally the case with photographic film, where silver platelets are formed at locations of high exposure upon development.) These partially reflecting mirrors transmit part of the playback wave along its direction of incidence, while deflecting the remaining part along an angle $-\phi$ with the $z$ axis, in accordance with the law of reflection. Now, for these reflected waves to interfere constructively and recreate the original object wave, the optical path-length (or phase) difference between reflections from adjacent fringe planes must be precisely one wavelength (or its integer multiples). Simple trigonometry reveals that this requirement will be met if the playback angle satisfies the condition

$$
\sin \phi=\frac{\lambda}{2 \Lambda}=\sin \theta \Rightarrow \phi_{B}=\theta .
$$

Here $\phi_{B}$ is referred to as the Bragg angle, and this particular playback wave, designated as $\bar{k}_{B}$, is said to be Bragg-matched to the grating. Evidently, the playback wave is scattered by the grating in such a way that the diffracted wave vector satisfies $\bar{k}_{D}=\bar{k}_{b}-\bar{k}_{C}$, thus closing the reconstruction wavevector diagram (conservation of momentum). Note that the Bragg condition is also satisfied for $\phi=-\theta$, which is the case of object wave reconstructing the reference wave, as well as for $\phi= \pm(\pi-\theta)$ (i.e., from right to left in Fig. $2 b$ ) corresponding to the cases of conjugate object wave reconstructing the conjugate reference wave and vice versa.

It should be evident, even from this simplistic description, that as the scattering of the playback wave starts giving rise to the original object wave inside the medium, this wave itself gets scattered by the grating, coupling its energy back into the play. back wave. There is, in fact, a steady exchange of energy (or "multiple reflections") between these two waves as they co-propagate through the grating - a process known as nwo-wave mixing. Therefore, the diffraction efficiency $\eta$ of the grating, defined as the ratio of the first-order diffracted power to the incident power, may be expected to depend on the optical interaction distance $n_{1} d / \cos \theta$ in a periodic fashion, and a complete power transfer between the two waves (i.e., $\eta=1$ ) should be feasible. In addition, we may expect a Bragg-mismatched playback wave to lose some of its power to higher-order grating modes (with wave vectors $\bar{k}_{n}=\bar{k}_{B}-n \bar{k}_{G}, n=\ldots,-2,-1,2$, $3, \ldots$ ), yielding only a partial reconstruction (i.e., $\eta<1$ ). This problem of power loss to higher orders is also encountered with gratings that are nonuniform (i.e., decaying in modulation into the depth of the medium) due to the ever-present absorption, or nonsinusoidal (i.e., over- and under-exposed at their extrema, or "saturated" and "cut off") due to the typically nonlinear recording dynamics of the material.

This intuitive picture of volume diffraction was substantiated formally in a seminal paper published by $\mathrm{H}$. Kogelnik in 1969, where an approximate yet highly satisfactory coupled-wave approach was developed to solve the scalar Helmholtz equation $\nabla^{2} U(\bar{r})+k^{2} n^{2}(\vec{r}) U(\bar{r})=0$ for the total optical field $U$ inside the grating. Kogelnik's analysis shows that the diffraction efficiency of a thick sinusoidal phase grating can be expressed as

$\eta=\frac{\sin ^{2} \Psi \sqrt{1+\Omega^{2}}}{1+\Omega^{2}}, \quad \Psi \equiv \frac{\pi n_{1} d}{\lambda \cos \theta}, \quad \Omega \equiv \frac{\sin 2 \theta}{n_{1}} \Delta \theta$,

where $\Delta \theta$ is the angular desuning of the playback wave from the Bragg angle $\theta$. The dependence of $\eta$ on $\Delta \theta$ is plotted in Fig. $2 c$, where we firstly observe a broad main lobe: essentially, the finite size (in our case thickness) of the medium has the net effect of spreading the grating angular ( $\bar{k}$-space) spectrum into a range of wave vectors centered at $\vec{k}_{\dot{c}}$. One can therefore visualize a cloud of grating vectors around the tip of $\bar{k}_{i}$ in $\bar{k}$ space (position-momentum un- 
certainty), the consequence being that the Bragg condition can now be (at least partially) satisfied by a range of playback waves $\bar{k}_{l} \neq \vec{k}_{H}$ that may not be perfectly Bragg-matched to the grating (Fig. $2 b$ ). We secondly note the appearance of the so-called Bragg nulls, the first of which occurs for a $\Delta \theta$ value of approximately $\Theta \equiv \lambda / 2 d \sin \theta$ : there is a discrete set of roughly equally spaced reconstruction angles at which no grating diffraction is observed. This suggests the possibility of recording many holograms within the same physical volume by using reference waves at angles (or "addresses") $\theta_{0} \pm n \Theta, n=0,1,2$, $\ldots$, around some nominal center angle $\theta_{0}-$ a scheme known as angular multiplexing. Since each hologram sits at a Bragg null with respect to all the other holograms, it should thus be possible to reconstruct individual holograms without any interference from the brhers. In practice, of course, recorded object patterns have some spatial structure (representing the information being stored) with a corresponding spread in their angular spectra, and therefore some cross talk between retrieved pattems is inevitable.

As the angular bandwidth $\Theta$ of a thick grating is inversely proportional to its width, it would seem that the thicker the medium can be made, the higher the attainable storage density becomes, and in fact storage of several thousand angle-multiplexed holograms has been routinely demonstrated in recent experiments. The ultimate physical limit on the storage density of a medium therefore comes from its finite dynamic range: each recorded hologram uses up a certain portion of the total available refractive-index change, and once the entire range is exhausted, no more holograms can be recorded even if the spatial bandwidth of the medium would allow it. (For a large number $N$ of multiplexed holograms, the average diffraction efficiency per hologram has been found empirically to scale as $1 / N^{2}$.) It may also be worthwhile to note here that in multiplexed recording, holograms far apart in recording order experience notably different exposure conditions due to the changing optical properties of the medium. It is therefore imperative that an optimal exposure schedule be formulated for the particular storage material being used to obtain equal diffraction efficiencies for all of the $N$ holograms.

Finally, mention should also be made of other multiplexing schemes that can achieve similarly dense holographic storage. For instance, the kind of Bragg detuning described above can also be achieved by holding the reference angle fixed and instead changing the wavelength - a scheme referred to as wavelength multiplexing. In an alternative technique known as phase-code multiplexing, reference waves are chosen from a set of orthogonal (2-D) phase dis- tributions. Yet another method that has been studied vigorously in recent years is shift multiplexing, where a highly divergent spherical beam is used as reference, and detuning is achieved by slight lateral translation of the medium. Depending on the affordable level of system complexity, any one or a combination of these and other (e.g., speckle, fractal, peristrophic, etc.) multiplexing techniques may be used.

\section{Storage .Materials}

As can be inferred from the foregoing discussion, the characteristics of the recording material are of paramount importance for volume holographic applications. A list of ideal physical attributes for a holographic storage medium may include the following:

- Recording mechanism - a large dynamic range of optically induced, and preferably optically erasable, refractive-index change (e.g., $\Delta n_{\max } \cong$ $10^{-3}$ to $\left.10^{-2}\right)$, negligible absorption;

- Sensitivity - responsive to (widely and cheaply available) red wavelengths, an appreciable holographic writing sensitivity (e.g., on the order of $10^{-2} \mathrm{~cm}^{3} \mathrm{~J}$ ) requiring low recording powers;

- Optical quality - suitable for casting in the form of thick slabs with large surface areas (i.e., a thick disk), high resolution (e.g., up to $5000 \mathrm{cy}$ cles/mm), negligible scattering;

- Stability - retain recorded data indefinitely over a wide range of ambient (temperature, humidity, etc.) conditions, show low fatigue over many (e.g., millions of) write-read-erase cycles;

- Volatilin - a (simple) physical means of "fixing" the recorded holograms so that they are not weakened (or erased) by subsequent recording and read-out beams;

- Self-processing - no need for processing or developing of any kind (e.g., chemical, thermal, magnetic, UV, IR, etc.) before or after recording; and last but not least,

- Cost - material readily and cheaply available or manufacturable.

Although photographic silver-halide emulsions have been the work horse of traditional holography, they fail to meet many of these requirements, and a host of more suitable materials has been found and developed for holographic storage. None of the candidate holographic storage media considered so far, however, has been able to fulfill all the requirements, and instead of a single "magical" material, an arsenal of possible materials, each with a unique set of strengths and weaknesses has emerged. Among these are photopolymer films (available from DuPont and 
Pularoid), photorefractive crystals such as iron-doped lithium-niobate ( $\mathrm{Fe}: \mathrm{LiNbO}_{3}$ ), and photochromic films such as those made from dichromated gelatin and the light-harvesting protein BacterioRhodopsin. For a given type of memory to be developed, it is thus likely that a sufficiently suitable material can be found among this collection, and the storage system can then be designed to compensate for the shortcomings of the material to the extent possible.

The key point of departure between different holographic materials is the nature of the physical recording process, which largely determines most of the other properties of the storage medium. For instance, in an impurity-doped electro-optic oxide like $\mathrm{Fe}: \mathrm{LiNbO}_{3}$, an inhomogeneous space-charge distribution is created inside the medium via the diffusion of electron-hole pairs excited by the illuminating intensity, and the associated electric field then locally modingtes the refractive index of the medium via the linear electro-optic effect. In a photochromic medium like a BR film, meanwhile, the incident intensity creates a spatially varying volume population difference between the two stable states of the molecule, which leads directly to an absorption modulation that is necessarily accompanied by a refractiveindex change through the Kramers-Kronig relation (statement of causality). Both of these materials are optically erasable and hence suitable for use in a ReWritable memory design (despite their low sensitivity); however, this very property also leads to volatility, requiring often complex engineering solutions (e.g., two-photon gated recording, thermal or electrical fixing, etc.) for data persistence. On the other hand, refractive-index changes can also be induced in (organic) photopolymers by polymerizing a monomer with visible illumination. Since these materials typically offer a considerably larger dynamic range, they are definitely a more attractive option for a Write-Qnce Read-Many type of memory where their irreversibility and low sensitivity are of little concern.

\section{System Architectures}

The components that comprise a typical holographic optical data storage system are

- a coherent source (array) or collection of sources that provide object, reference, and reconstruction waves, and possibly another source for erasure;

- a Spatial Light Modulator for preparing the (binary or multi-level) data to be stored as 2-D images (or "pages");

- a detector (array) and subsequent electronics for data read-out, post-detection signal processing, and error correction;
- optics for routing and imaging the wavefields within the system, along with other components for performing data multiplexing; and finally

- a storage medium within which holograms may be written by altering the optical properties of the material through some physical process.

A page-oriented holographic optical memory architecture featuring these components is depicted in Figure 3, which is the $90^{\circ}$-geometry commonly used with photorefractive crystals to achieve maximum angular selectivity. A pair of high-quality lenses forms a $+-f$ imaging system that matches the pixels of a (2-D) SLM to those of a CCD camera or a CMOS detector array, and the crystal is placed at the Fourier plane of this setup. During recording, data is composed as a binary or gray-level image on the SLM and subsequently impressed on a collimated object beam, whose Fourier transform is then formed inside the crystal by lens $L_{1}$. At the same time, a plane reference wave is introduced from the side of the crystal at a unique angle designated for that data page, thus recording a Fourier hologram inside the crystal. During retrieval, this page is addressed at the same reference angle and the diffracted field is (inverse) Fourier-transformed by lens $L_{2}$, thus forming the image of the original data page on the detector. Due to the high angular selectivity of the medium, many pages can be multiplexed within the crystal volume and randomly accessed by use of the appropriate addressing reference beams. This page-oriented data storage scheme also facilitates parallel data transfer, thus enabling potentially very high read-out rates.

The design of a holographic data storage system starts with the specification of a raw Bit-Error Rate based on a target user BER and an affordable ErrorCorrection Coding scheme of choice: typically, an acceptable BER of $10^{-12}$ can be delivered to the user with a reasonable ECC overhead if a raw BER of $10^{-4}$ can be attained at the detector. This, in turn, translates into a minimum Signal-to-Noise Ratio that must be achieved by the system at its output. Among the numerous and inter-related factors determining the SNR are source wavelength and power, medium dynamic range, thickness, diffraction efficiency, and scattering, inter-page and inter-pixel cross talk determined by the number of multiplexed pages, number of bits per page, and the imaging system point-spread function, detector integration time and electrical noise, and other detrimental influences such as misalignments and nonuniformities.

Due to the difficulties involved in working with photorefractive crystals and the pressures placed on the research community to produce a commercially viable technology, increasing attention has also been paid to a holographic disk paradigm. Such a system 


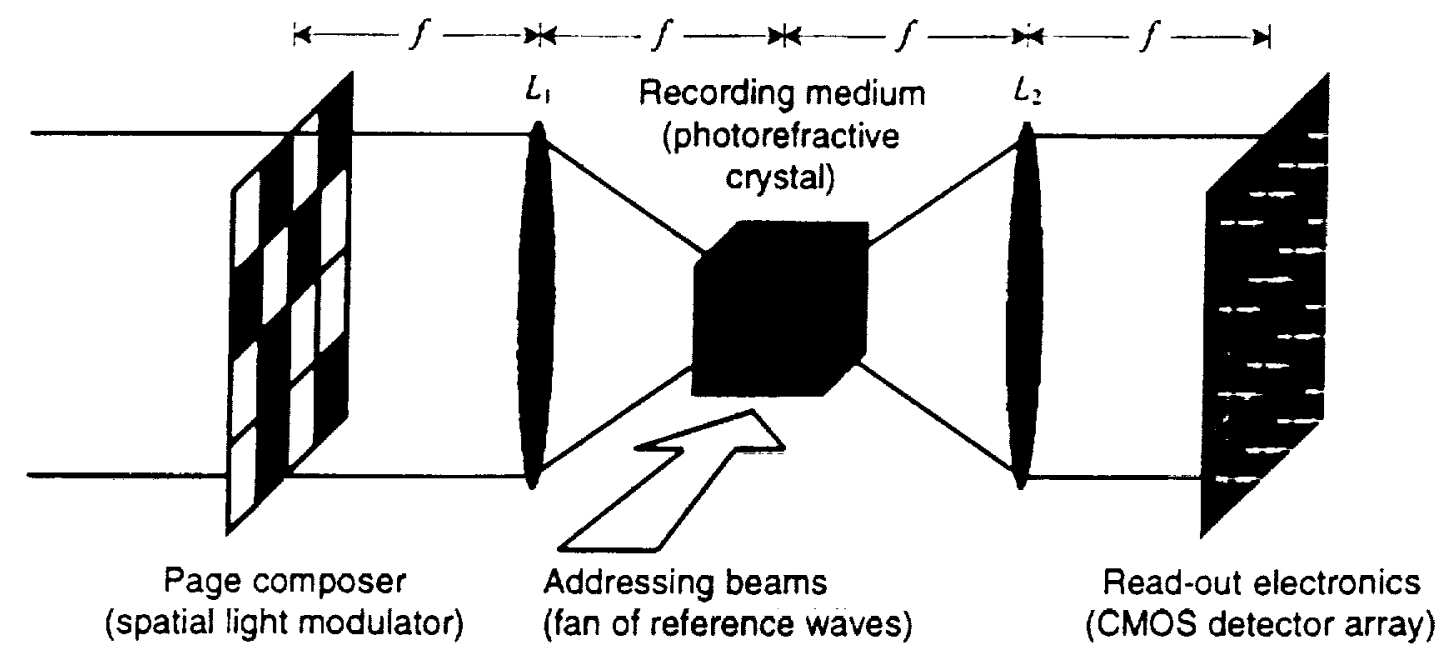

Figure 3 The standard holographic optical data storage system architecture

$\rightarrow$

may employ a thick photorefractive organic-polymer disk with a spiral single- or multi-track data format (much like the familiar CD/DVD technology) that is accessed holographically by shift, speckle, or phasecode multiplexing. In recent years, teams at universities (California Institute of Technology, Stanford University), government and industry research laboratories (IBM Almaden Research Center, Lucent Technologies - Bell Laboratories, NASA), and small companies (Siros Technologies, Holoplex, Inc.) have been actively pursuing the optical head, media, and system design for commercial WORM and RW holographic optical data storage products. The present goal is to manufacture a system capable of a storage capacity of about $50 \mathrm{~GB}$, with roughly $100-\mathrm{ms}$ re-

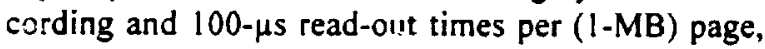
which may fulfill the market need for a memory that is cheaper than silicon DRAM while offering faster access than magnetic storage.

There is a rapidly increasing demand for highcapacity and fast-access data storage in virtually all avenues of human endeavor from medicine and education to business and communications, from multimedia and entertainment to military and space. With the development of suitable architectures and materials, and the cost-effective availability of enabling technologies, holographic storage is well positioned to satisfy this need in the near future.

\section{Authors}

Doğan A. Timuçin is a research scientist in the lnformation Physics Group at NASA Ames Research Center, Moffett Field, CA, where he has been since 1995. He received the B.S., M.S., and Ph.D. degrees in Electrical Engineering from the Middle East Tech- nical University in 1989 and Texas Tech University in 1991 and 1994, respectively. His current research interests are in holographic optical data storage (particularly with bacteriorhodopsin films), near-field optics, quantum optoelectronics, and quantum information processing.

John D. Downie is a research scientist in the Optical Network Research Department at Corning, Inc., Sullivan Park Science and Technology Center, Corning, NY. He received a B.S. in Optics from the University of Rochester in 1985 and a Ph.D. in Electrical Engineering from Stanford University in 1989. He has worked at both NASA Ames Research Center and Lawrence Livermore National Laboratory before joining Corning in 1999. His current reseaich is centered on optical communications, optical network architectures, and optical network performance monitoring.

\section{Further Reading}

- J. F. Heanue, M. C. Bashaw, and L. Hesselink, "Volume holographic storage and retrieval of digital data," Science 265, 749-752 (1994).

- J. W. Goodman, Introduction to Fourier Optics, 2nd ed. (McGraw-Hill, New York, 1996).

- P. Hariharan, Optical Holography - Principles, Techniques, and Applications, 2nd ed. (Cambridge University Press, Cambridge, UK, 1996).

- R. J. Collier, C. B. Burckhardt, and L. H. Lin, Optical Holography (Academic Press, New York, 1971).

- G. T. Sincerbox, ed., Selected Papers on Holographic Storage (SPIE Milestone Series, Vol. MS95, Bellingham, WA, 1994). 